\title{
Clear-PEM system counting rates: a Monte Carlo study
}

\author{
P. Rodrigues, ${ }^{a}$ A. Trindade ${ }^{a *}$ and J. Varela ${ }^{a b c}$ \\ ${ }^{a}$ Laboratório de Instrumentação e Fìsica Experimental de Partìculas (LIP), \\ Av. Elias Garcia 14-1 1000-149 Lisboa, Portugal \\ ${ }^{b}$ Instituto Superior Técnico (IST) da Universidade Técnica de Lisboa, \\ Av. Rovisco Pais 1049-001 Lisboa, Portugal \\ ${ }^{c}$ European Organization for Nuclear Research (CERN), \\ CH-1211 Genève 23, Switzerland \\ E-mail: andreia@lip.pt
}

Abstract: Positron Emission Mammography (PEM) with ${ }^{18}$ F-Fluorodeoxyglucose $\left({ }^{18} \mathrm{~F}\right.$-FDG) is a functional imaging technique for breast cancer detection. The development of dedicated imaging systems with high sensitivity and spatial resolution are crucial for early breast cancer diagnosis and an efficient therapy. Clear-PEM is a dual planar scanner designed for high-resolution breast cancer imaging under development by the Portuguese PET Mammography consortium within the Crystal Clear Collaboration. It brings together a favorable combination of high-density scintillator crystals coupled to compact photodetectors, arranged in a double readout scheme capable of providing depth-of-interaction information.

A Monte Carlo study of the Clear-PEM system counting rates is presented in this paper. Hypothetical breast exam scenarios were simulated to estimate the single event rates, true and random coincidence rates. A realistic description of the patient and detector geometry, radiation environment, physics and instrumentation factors was adopted in this work. Special attention was given to the ${ }^{18}$ F-FDG accumulation in the patient torso organs which, for the Clear-PEM scanner, represent significant activity outside the field-of-view (FOV) contributing to an increase of singles, randoms and scattered coincidences affecting the overall system performance. The potential benefits of patient shielding to minimize the influence of the out-of-field background was explored. The influence of LYSO:Ce crystal intrinsic natural activity due to the presence of the ${ }^{176} \mathrm{Lu}$ isotope on the counting rate performance of the proposed scanner, was also investigated.

KEYWORDS: Gamma camera, SPECT, PET PET/CT, coronary CT angiography (CTA); X-ray mammography and scinto- and MRI-mammography.

${ }^{*}$ Corresponding author. 


\section{Contents}

1. The Clear-PEM scanner 1

2. Monte Carlo simulation 3

2.1 The patient model

2.2 The detector model

2.3 Breast exam scenarios

3. Counting rates results 7

3.1 Front-end count rates

3.2 Expected coincidence rates 8

3.3 Effect of ${ }^{176} \mathrm{Lu}$ natural background radiation 12

4. Discussion and conclusions 14

\section{The Clear-PEM scanner}

Positron Emission Tomography (PET) with ${ }^{18} \mathrm{~F}$-Fluorodeoxyglucose $\left({ }^{18} \mathrm{~F}\right.$-FDG) is a functional imaging technique that has been proposed as a complementary technique to X-ray mammography in the diagnosis and treatment monitoring of breast cancer [1]. In the recent years, significant progresses has been made in the development of compact systems for breast imaging known as Positron Emission Mammography (PEM) scanners [2, 3].

Clear-PEM is a dual planar scanner designed for high-resolution breast cancer imaging. The scanner is being developed by the Portuguese PET Mammography consortium, within the framework of the Crystal Clear Collaboration [ $[$. It brings together a favorable combination of high-density scintillator crystals coupled to compact photodetectors, arranged in a double readout scheme capable of providing depth-of-interaction (DOI) information [5]. Such characteristics could significantly improve breast imaging without sacrificing neither the sensitivity nor the spatial resolution of the system [6, 7]. Experimental measurements has already demonstrated that Clear-PEM detector modules have adequate performance, achieving in particular a measurement of the photon interaction point along the crystal length with $2 \mathrm{~mm}$ FWHM resolution. The peak detection sensitivity was found to be $6.6 \%$ (without trigger simulation) when the detection heads are separated by $10 \mathrm{~cm}$. In addition, the reconstructed image of a simulated point source located at the center of the FOV indicates a spatial resolution of $1.4 \mathrm{~mm}$ FWHM (transaxial and axial resolution) [D]. The detector will be housed in a mechanical gantry that allows to rotate it around the breast for tomographic reconstruction. This represents an advantage over stationary planar detectors in terms of the quality of the reconstructed images. The dual planar geometry of the Clear-PEM detector is rather flexible and can easily accommodate different breast shapes and sizes by simply positioning 


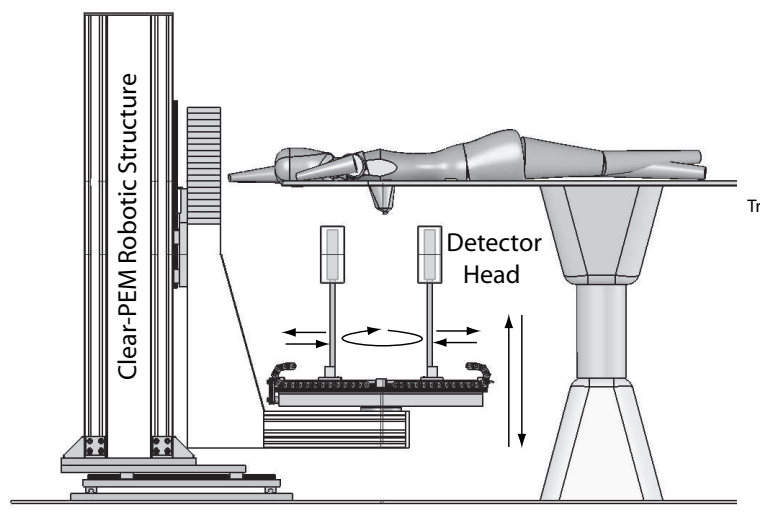

(a)

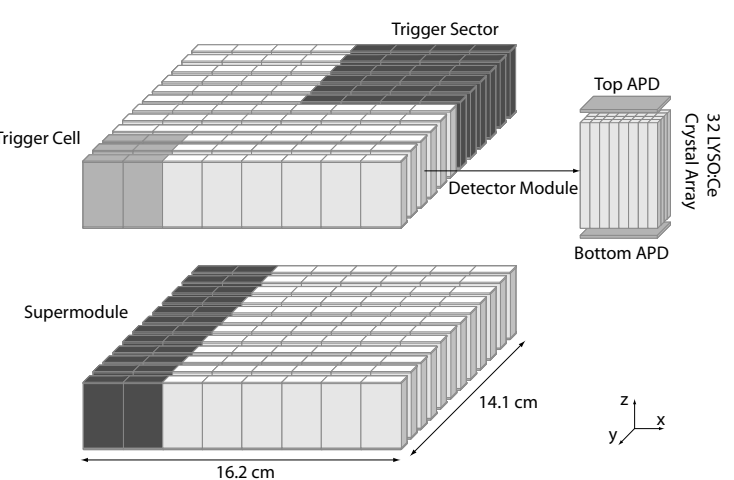

(b)

Figure 1. (a) Representation of a breast exam with the Clear-PEM detector. (b) Schematic drawing of the Clear-PEM scanner.

the detectors at different separation distances, while allowing to apply lateral compression of the breast during the exam - figure 1(a), Breast compression improves lesion detectability by increasing the detection sensitivity and contrast by reducing the scattered background. It also prevents the patient movement that can result in image artifacts.

The Clear-PEM scanner consists of two parallel detector heads with approximately $16 \times 14 \mathrm{~cm}^{2}$, each one holding 96 detector modules. One module is an array of $4 \times 8$ LYSO:Ce crystal pixels with dimensions of $2 \times 2 \times 20 \mathrm{~mm}^{3}$. The readout of each module is performed by two 32-pixel Hamamatsu S8550-01 avalanche photodiodes (APDs), optically coupled on each side of the crystal array. Twenty four modules are grouped $(2 \times 12$ configuration $)$ in a mechanical structure called supermodule with about $4 \times 14 \mathrm{~cm}^{2}$. The final assembling of one detector head groups four supermodules side by side - figure 1(b). This leads to a total of 6144 LYSO:Ce crystals. The estimated packing fraction of the detector defined as the volume occupied by the scintillation crystals is 52\%. Groups of six APDs are connected to low noise amplifier and multiplexer ASICs that perform the readout, amplification, sampling and storage in analogue memories of 192 channels (trigger cell). The ASICs also perform the selection of the two active channels (multiplexing of 192:2) above a common threshold [8]. The output analogue sampled pulses are digitized by 10bit sampling ADCs (Analogue Digital Converter), serialized in LVDS (Low-Voltage Differential Signaling) bit streams and transmitted to the off-detector system. At this level, four DAQ boards (8 FPGAs) are responsible for the data reduction and pipeline data storage. Parallel algorithmic processing is used to minimize dead time while extracting the amplitude and time from the detector pulses [8]. Each DAQ Board FPGA processes information from four trigger cells (trigger sector) that corresponds to a cluster of 768 LYSO:Ce crystals. Data is sent to a trigger processor (TGR/DCC Board), which selects two-photon events in coincidence within a programmable timing window. At each trigger, the relevant dataframes are transmitted to the acquisition PC for further analysis [9]. The DOI coordinate within the crystal where the interaction took place is estimated from the asymmetry of the collected light at the top and bottom APD pixels. For those events with 
more than one active crystal due to Compton scattering, an event reconstruction algorithm combining the position and energy information is used to assign the coordinates of the first interaction [10]. The final coordinates within the crystal using the interaction depth information are used to define lines-of-response (LORs) for those events within the selected energy window. The validated LORs are stored in a list-mode file to initiate the image reconstruction process [5].

Monte Carlo simulations carried out to evaluate the count rate performance of the Clear-PEM scanner, are presented in this paper. The single event rates, true and random coincidence rates were estimated under representative breast exam scenarios, carefully selected to establish the baseline operational conditions for the scanner. The noise equivalent count (NEC) rate metric was used to characterize the quality of acquired data in each case-study. The ${ }^{18}$ F-FDG accumulation in other organs, representing a significant portion of background activity located outside the FOV of the scanner, is an important question of concern in Positron Emission Mammography. Events originating outside the FOV will increase detected singles, randoms and scattered coincidences. Single events may affect the system data acquisition performance, introducing undesirable dead time and loss of efficiency, while randoms and scatters will be responsible for the degradation of the final image quality. Therefore, the potential benefits of patient shielding to minimize the influence of the out-of-field background in the Clear-PEM scanner, was also investigated. In addition, the effect of LYSO:Ce crystal intrinsic radioactivity due to the presence of the ${ }^{176} \mathrm{Lu}$ isotope in the overall performance, was also assessed and its influence in the image quality index discussed.

\section{Monte Carlo simulation}

In the adopted simulation methodology, efforts have been made in order to reproduce the radiation environment, physics and instrumentation factors as realistically as possible, which guarantees that results are of practical value for the scanner development phase. That requires an anthropomorphic patient phantom to simulate the background activity, besides the detailed description of the detector geometry and an effective modeling of the data acquisition and trigger systems. These components were integrated in a dedicated Monte Carlo simulation framework specially developed for the Clear-PEM scanner, based on the Geant4 toolkit [11, 12]. The study conditions are described in the next sections.

\subsection{The patient model}

The NURBS CArdiac Torso (NCAT) phantom, provides a realistic anthropomorphic model of the human anatomy suitable for nuclear medicine imaging applications [13]. In addition, it include breast models that allows to reproduce the prone and supine exam positions, of interest for the purposes of this work. A set of parameters are available to control the shape of the breast in the phantom geometry. The NCAT phantom was used to generate three female patients (A, B and C) with different breast shapes and volumes. The dimensions of the body organs (heart, liver, kidneys, lungs, spleen and stomach) and bone structures (spine and ribs) were set to the default values derived from the 3D Visible Human dataset [14] - figure 2. It is well known that organs with higher uptakes and close to the breast, like the heart, will play an important role in generating the out-of-field background [15]. In particular, the heart takes a special importance when the left breast is under imaging [16]. Such an examination scenario is expected to be the most challenging 


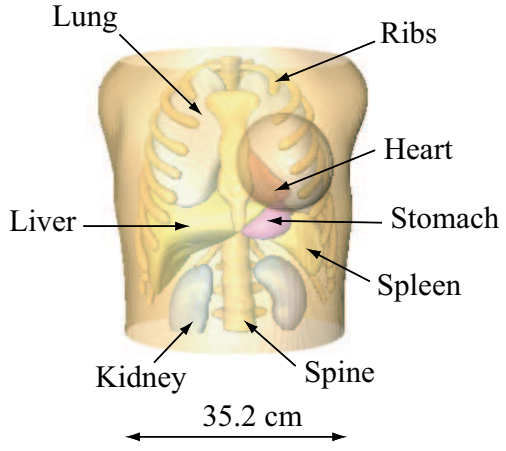

(a)

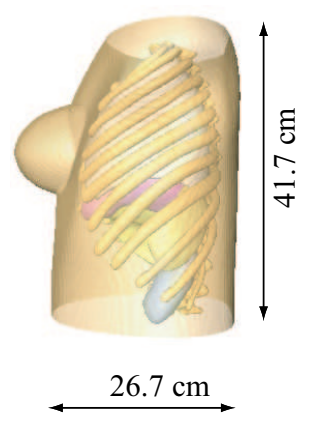

(b)

Figure 2. Volume renderings of the NCAT phantom voxelized geometry. Different organ models and bone structures are shown. Front (a) and lateral (b) views with the indication of the torso dimensions.

for Positron Emission Mammography scanners and so it was chosen for the Clear-PEM simulation studies presented in this paper. The left breasts of patient $\mathrm{A}$ and $\mathrm{B}$ mimic two uncompressed models approximating a small $\left(600 \mathrm{~cm}^{3}\right)$ and a medium/large $\left(1200 \mathrm{~cm}^{3}\right)$ breast. From patient $\mathrm{B}$ to patient $\mathrm{C}$, the breast volume was kept constant but the shape parameters were adjusted to reproduce a moderate breast compression with a slightly lateral deformation. Each phantom was generated with a total of $128 \times 128 \times 128$ cubic voxels each one with a resolution of $0.325 \mathrm{~cm}^{3}$. A voxel identification index was used to retrieve, on the fly, the material properties and radiotracer concentration values associated to a given organ/tissue. This allows to generate $3 \mathrm{D}$ spatial distributions of ${ }^{18} \mathrm{~F}$ FDG radiotracer according to each organ particular shape. The densities and chemical composition of the biological tissues for the calculation of electromagnetic interaction cross-sections, were extracted from the International Commission on Radiation Units and Measurements (ICRU) Report 46 [17] and included into the Geant 4 material database. In order to reproduce the radiation environment present in a real scan, the background from all organs within the torso must be taken into account. Besides the heart, activity distributions for the liver, kidneys, lungs, spleen, stomach and adipose tissue filling the body contour were also considered. In the present work, standardized uptake values (SUV) for normal tissues available in the literature were used to compute the 18F-FDG concentrations for the various organs in the NCAT phantom [18]. The SUVs are representative of uptake values measured 60 minutes after an injection of 300-370 MBq. An average body weight of $75 \mathrm{~kg}$ and a typical injected dose of $370 \mathrm{MBq}$ for PET exams was assumed in the calculation of the $18 \mathrm{~F}-\mathrm{FDG}$ concentrations shown in table 1 . The tissue concentration values were used to compute the mean number of ${ }^{18} \mathrm{~F}$ radioactive decays inside each voxel of the phantom, during a simulation run. The tracking of each individual annihilation photon was performed throughout the phantom geometry until it reaches the scoring volume, considered to be the air surrounding the patient, where particles are stored for later tracking. Annihilation photons, that have undergone Compton interactions within the body tissue, were tagged in order to distinguish the scattered from unscattered events after the trigger simulation. The relevant physical processes from the Geant4 Low Energy Electromagnetic package over the energy range of interest were considered for the tracking of photons, electrons and positrons. For photons, Rayleigh scatter, Compton scatter, photoelectric absorption with atomic de-excitation due to characteristic X-rays or Auger electrons were 


\begin{tabular}{|c|c|c|}
\hline Organ & $\mathbf{S U V}_{\mathrm{avg}} \pm$ s.d. $\left(\right.$ g.mL $\left.\mathbf{m}^{-1}\right)$ & FDG Conc. \pm s.d. $\left(\mathrm{kBq} \cdot \mathrm{mL}^{-1}\right)$ \\
\hline Body and breast & $0.43 \pm 0.21$ & $2.12 \pm 1.04$ \\
\hline Heart blood-pool & $1.42 \pm 0.33$ & $7.01 \pm 1.63$ \\
\hline Heart myocardium & $4.44 \pm 2.50$ & $21.90 \pm 12.33$ \\
\hline Liver & $1.86 \pm 0.27$ & $9.18 \pm 1.30$ \\
\hline Kidneys & $1.74 \pm 0.39$ & $8.58 \pm 1.92$ \\
\hline Lungs & $0.40 \pm 0.06$ & $1.97 \pm 0.30$ \\
\hline Spleen & $1.26 \pm 0.23$ & $6.22 \pm 1.13$ \\
\hline Stomach & $1.99 \pm 0.53$ & $9.82 \pm 2.61$ \\
\hline
\end{tabular}

Table 1. Standardized uptake values and corresponding 18F-FDG concentrations for the various organs of the NCAT phantom. The 18F-FDG concentration for each tissue was computed as the standard uptake value multiplied by a typical injected activity of $370 \mathrm{MBq}$ and divided by an average body weight of $75 \mathrm{~kg}$.

considered. For positrons and electrons the multiple Coulomb scattering, discrete ionization and bremsstrahlung were taken into account. The standard Geant 4 positron annihilation process was replaced by a modified one that allows the simulation of non-collinear photon emission due to thermal electron movement. Particles were tracked down to $250 \mathrm{eV}$ and the range cut for the production of secondary particles was set to $10 \mu \mathrm{m}$, for all materials in the phantom. The corresponding energy cutoffs for electrons are $6.2 \mathrm{keV}$ for adipose tissue, $10.2 \mathrm{keV}$ for the bone and $990 \mathrm{eV}$ for lungs. For photons, it corresponds to an energy cutoff of $990 \mathrm{eV}$ in all biological materials.

\subsection{The detector model}

The relevant tracking information of the stored annihilation photons leaving the phantom geometry are transfered to the detector simulation module. This module implements in full detail the detector geometry, including the scintillation crystals and their wrapping, avalanche photodiodes, optical coupling and all the front-end electronic components. Photons and their secondary particles that reach the detector heads are tracked throughout the geometry taking into account Compton and photoelectric interactions within the different materials. The deposited energy together with the crystal identification are then fed into the data acquisition and trigger simulation module.

The response of the DAQ/Trigger system was also incorporated into the simulation studies. However, the number of exam scenarios and the required statistics did not allow us to perform a detailed simulation of the complete readout chain [0]. As an alternative, a parameterization describing the trigger system behavior was established. Transfer functions for the DAQ Board efficiencies, energy resolution in the on-line trigger $(\Delta \mathrm{E} / \mathrm{E}=16 \%$ at $511 \mathrm{keV})$ and single photon time resolution $(\sigma=1.45 \mathrm{~ns}$ at $511 \mathrm{keV})$ as function of the deposited energy in the crystals, for the baseline configuration (50 MHz system frequency) of the Clear-PEM scanner were implemented in this module [9]. The fast trigger module allows the selection of the energy thresholds, the time windows of the data acquisition electronics, and the duration of the ASIC readout cycle for each simulation setup. In the present studies a specific combination of parameter settings was used table 2. An energy threshold of $150 \mathrm{keV}$ and a quite large coincidence window of $10 \mathrm{~ns}$ were selected in order to allow a more refined off-line data analysis as a function of these parameters. Such procedure aims to optimize the energy threshold and timing windows to be used by the ClearPEM scanner, in the different exam scenarios. 


\begin{tabular}{|l|c|}
\hline Front-end ASIC Common Threshold & $50 \mathrm{keV}$ \\
Front-end ASIC Readout Cycle & $250 \mathrm{~ns}$ \\
DAQ Board Compton Window & $20 \mathrm{~ns}$ \\
DAQ Board Compton Threshold & $50 \mathrm{keV}$ \\
DAQ Board Event Threshold & $150 \mathrm{keV}$ \\
Trigger Board Rejection Window & $16 \mathrm{~ns}$ \\
Trigger Board Coincidence Window $[-\tau, \tau]$ & $\tau=10 \mathrm{~ns}$ \\
\hline
\end{tabular}

Table 2. DAQ/Trigger system parameters adopted for the Clear-PEM performance simulation studies.

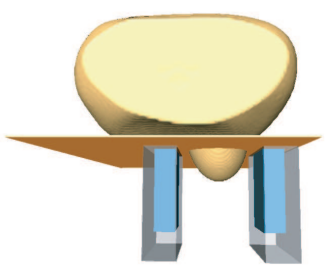

(a)

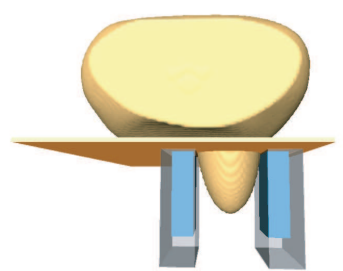

(b)

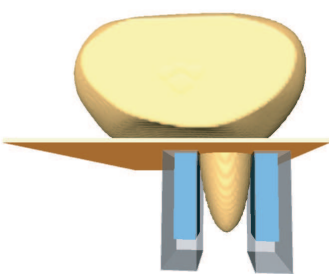

(c)

Figure 3. Visualization of the simulated imaging setup with shielding. (a) Patient A exam with the detector heads separated by $15 \mathrm{~cm}$. (b) Patient B exam with the detector heads positioned at $13 \mathrm{~cm}$. (c) Patient C exam with the detectors heads $10 \mathrm{~cm}$ apart. All separation distances are referred to the housing box of each detector head.

\subsection{Breast exam scenarios}

Hypothetical breast exams were simulated using the patient and detector response models previously described. For patient A, the left breast was imaged with the detector heads $15 \mathrm{~cm}$ apart. This first exam scenario tries to mimic the most challenging conditions for the prototype, because the separation distance between the detector heads was not optimized for the size of a small and uncompressed breast. For patient B, which has a medium/large breast, the exam was performed with the detector heads mounted $13 \mathrm{~cm}$ apart. This second exam conditions simulates what was considered to be the standard case, in which there was no need to compress the patient breast. In addition, the detector heads positioning procedure guarantees the maximum proximity to the target volume. The detector heads were adjusted to $10 \mathrm{~cm}$ separation distance to simulate the exam of patient $\mathrm{C}$, with a breast of medium/large size that have been moderately compressed. This exam scenario will allow to study the performance of the scanner in quite favorable imaging conditions and evaluate the advantages of breast compression. Note that all separation distances are referred to the housing box of each detector head. There is an additional space between the crystals front-face and the housing box, of about $2.5 \mathrm{~cm}$, to accommodate the front-end electronics and cooling system of the detector. The patient phantoms were all prone positioned with the detector heads at each side of their left breast - figure 3. A first set of simulations, for each exam scenario, were executed. The simulations were then repeated for the same cases, but with a shielding layer isolating the patient 


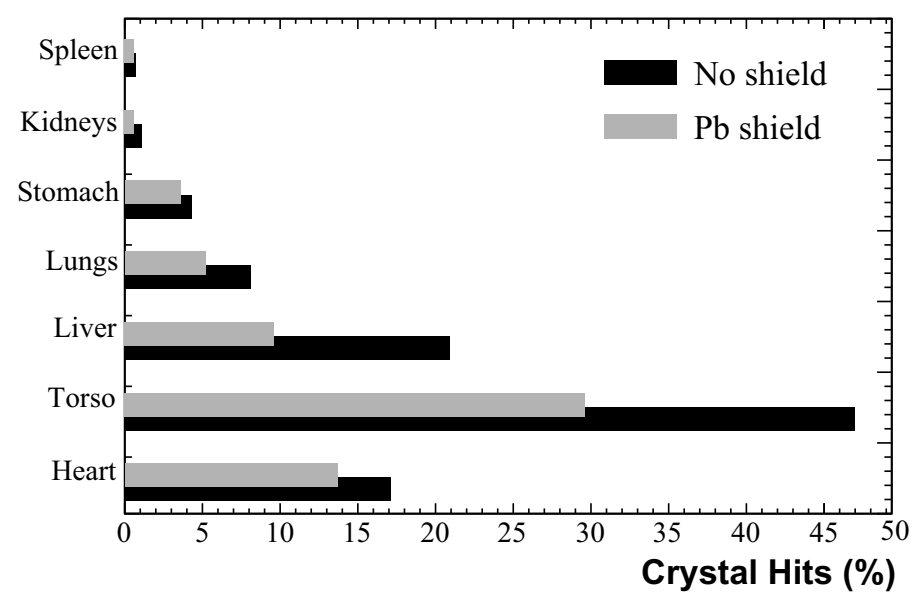

Figure 4. Individual organ contributions to the total number of photons above $5 \mathrm{keV}$ hitting the detector head for the simulation setups with and without shielding.

body from the detector heads, except for an aperture with $20 \times 20 \mathrm{~cm}^{2}$. This opening is required in order to allow the breast to be imaged. The shielding layer was made of lead $\left(\rho=11.4 \mathrm{~g} . \mathrm{cm}^{-3}\right.$ and $\mu=1.778 \mathrm{~cm}^{-1}$ ) with a thickness of $0.7 \mathrm{~cm}$ (70\% absorption for $511 \mathrm{keV}$ single photons) that adds to the examination table an extra weight of about $36 \mathrm{~kg}$. The shielding effectiveness on the reduction of out-of-field photon flux was assessed by comparing the results with and without the lead shield. Combining the phantom geometries and the possibility of shielding, six different exam scenarios have been simulated.

\section{Counting rates results}

\subsection{Front-end count rates}

Like other Positron Emission Mammography scanners, Clear-PEM was specifically designed to explore the breast anatomy by placing the detector heads in close proximity to the breast tissue during the exams. At the same time, the detector heads will be positioned very near the patient torso. Therefore, besides the activity from the breast, background activity from ${ }^{18}$ F-FDG radiotracer uptake in organs located outside the FOV will also contribute to the increase of the single events flux that reach the detector. The contribution of each organ to the total flux (crystal hits above $5 \mathrm{keV}$ ) was assessed for the three simulated exams. Results for patient A are shown in figure 4 . Similar conclusions can be drawn for patient $\mathrm{B}$ and $\mathrm{C}$. The major contributions were found to be from the torso $(47 \%)$, the liver $(21 \%)$ and the heart $(17 \%)$ that includes the myocardium and the blood-pool chambers. Although 18F-FDG concentration in the liver is two times lower than in the heart myocardium, the liver has a much larger volume yielding a higher activity and thus increasing its contribution to the total photon counts. For the same reason, the tissue filling the patient torso has such a significant contribution in spite of its low concentration value $(2.12$ $\mathrm{kBq} \cdot \mathrm{mL}^{-1}$ ). The contribution of all organs is reduced when the lead sheet is under the patient torso, and once again the major contributions were found to be from the same organs. However, the contribution from the liver is more reduced relatively to the heart. Because of the anatomical location of the heart, right behind the left breast, photons can more easily pass through the aperture 
in the lead. In turn, the liver remains almost completely shielded and the majority of photons coming from this organ are necessarily attenuated. The mean absorption for single events was found to be low, about $40 \%$, relative to the expected value. This is due to the fact that $57 \%$ of all singles that reach the detector front-end, actually pass through the aperture. The need for an aperture for the breast in the examination bed seems to compromise the shielding efficiency on the reduction of out-of-field background. It is important to point out that the relative organ contributions depend on which breast of the patient, left or right, is being imaged. For example, it is predictable that the liver contribution increases when a right breast examination is taking place, since the detector heads will be positioned closer to that organ. Such increase, will however be partially offset by the reduction of the heart background.

High front-end counting rates may cause an increase of the system dead time, which in turn impose longer examination times, or can even affect the overall operation of the imaging system. From the Clear-PEM operating point-of-view, the single rates per detector readout unit or trigger cell is the most critical issue. One trigger cell is read on both sides by two ASICs, one at the bottom and one at the top side of the detection head. The ASICs performs the amplification of the 192 crystals and select the two channels above the common threshold, that in this study was set at 50 $\mathrm{keV}$. The analog multiplexing of 192 to 2 channels restricts the readout of more than two channels above the selected threshold in the same time window, producing an error code and sending it to the off-detector electronics. Such error represents an ASIC overflow and, as a consequence, all collected data within one Front-end ASIC Readout Cycle of $250 \mathrm{~ns}$ is discarded. This option guarantees the integrity of the data captured by the off-detector FPGAs [9]. However, if the single rate per trigger cell is too high, the number of ASIC overflows increases, as well as the overall system dead time. In figure 5, the spatial distribution of single rates recorded in each trigger cell (16 in one detector head) for the exam scenario of patient $C$, is presented. In both configurations, with and without shielding, the trigger cells near the patient torso (located at $-80 \mathrm{~mm}$ x-axis) are exposed to a higher photon flux coming from outside the FOV than trigger cells in the opposite end of the detector. Besides that, there is a slightly difference between the single count rates in the inner and the outer detector heads. This is because the inner detector head is right in front of the patient chest, closer to the heart and liver, while the outer detector head is positioned near the patient arm, far away from large volume anatomical structures and organs with high ${ }^{18} \mathrm{~F}$-FDG uptakes. For the inner detector head and even in the presence of shielding, count rates above $350 \mathrm{kHz}$ are recorded. Those trigger cells may play an important role in the system dead time since the ASIC overflow probability for these readout units will be higher. At the opposite side of the detector head, single rates are significantly lower, around $60 \mathrm{kHz}$. The estimations for the system dead time due to the duration of the frontend ASIC readout cycle are below 5\% for all the exam scenarios. The presence of shielding does not offer an expressive advantage for realistic imaging conditions.

\subsection{Expected coincidence rates}

In order to evaluate the response of the Clear-PEM system when exposed to the simulated radiation environments, the true, scatter and random counting rates were assessed. The calculations were performed for different combinations of energy and timing windows. The lower energy threshold ranged from 150-450 keV and the timing window from 2-10 ns. The upper energy threshold was fixed at $700 \mathrm{keV}$ for all the combinations. Using the counting rates obtained in each study, NEC 


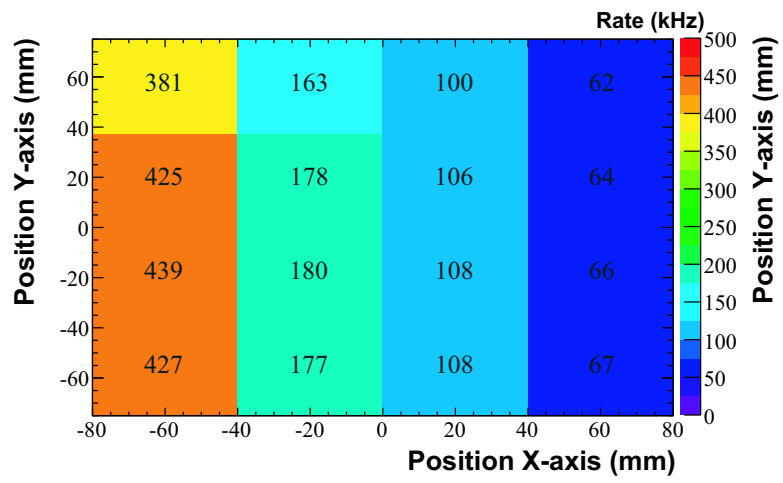

(a) No shield —inner detector head

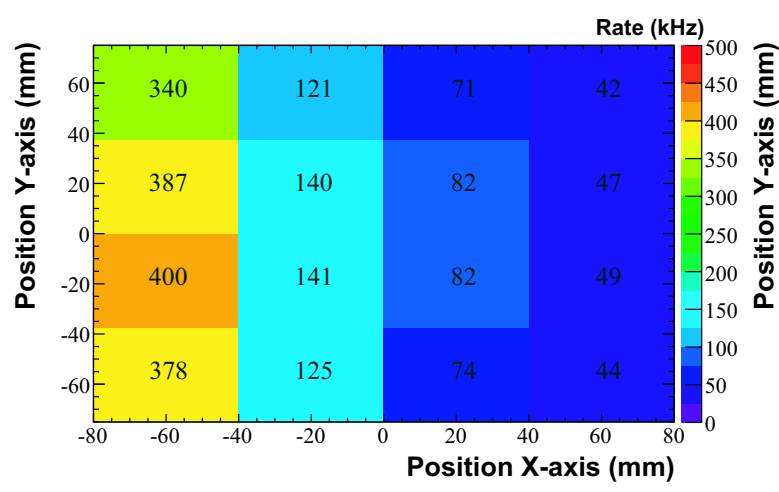

(c) $\mathrm{Pb}$ shield —inner detector head

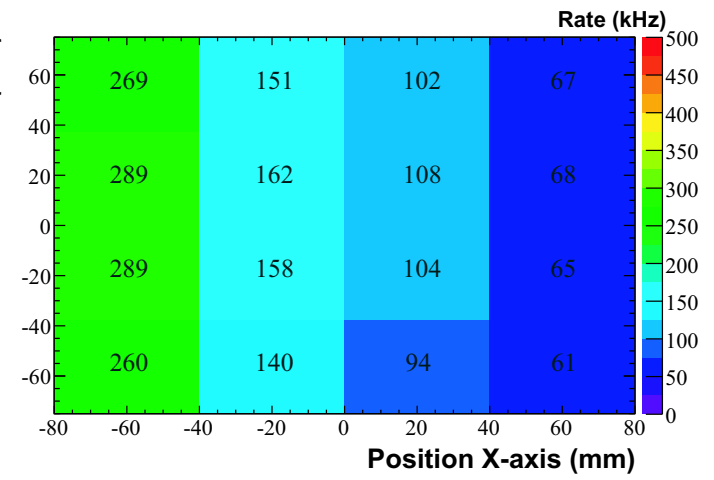

(b) No shield —outer detector head

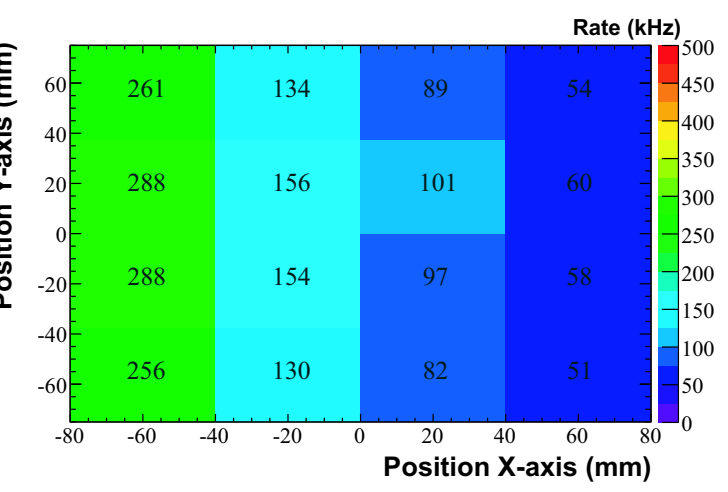

(d) Pb shield —outer detector head

Figure 5. Spatial distribution of single rates per trigger cell in both detection heads, with and without the presence of shielding, obtained from the patient $\mathrm{C}$ exam simulated data.

rate curves were also computed. The NEC metric provides an index of image quality based not only on the sensitivity to true events but also taking into account noise contribution from the random and scatter coincidences present in the acquired raw-data [19]. Usually NEC is calculated as a function of the radiotracer activity level, with the aim of optimizing the injected dose in the patient that maximize the quality of the data for a given acquisition. A different perspective was taken for the present study. The patient injected dose and radiotracer activities in the organs are specified and for that values, the time and energy windows are optimized within the pre-defined ranges. Noise equivalent count rate is defined as:

$$
N E C=\frac{T^{2}}{(T+S)+k f R}
$$

where, $T, S$ and $R$ are the total number of true unscattered coincidences, scattered events and random events detected within the FOV, respectively. Factor $k$ accounts for the increase of the random coincidences variance term due to the statistical noise introduced by the chosen method for randoms estimation. Since Clear-PEM will perform the randoms estimate using a delayed 
coincidence method, $k=2$ was assumed [19]. The statistical noise due to the random coincidences correction can eventually be reduced by smoothing the random data, which in the mammography case is not expected to present sharp variations. However in this study we do not consider this possible improvement. Factor $f$ is the fraction of random coincidences, from the total number of randoms in the FOV, defining LORs that pass through the object under imaging and will actually contribute to the noise in the final reconstructed image. For the present study, $f$ values were extracted directly from the Monte Carlo data. A fraction $f$ of 0.47 for patient A, 0.93 for patient $\mathrm{B}$ and 0.97 for patient $\mathrm{C}$ were found. The results as a function of the timing window and for a fixed energy window of 300-700 keV are presented in figure 6 .

The maximum true coincidence rates for patient A was found to be $1.2 \mathrm{kHz}$. The simulated breast volume $\left(600 \mathrm{~cm}^{3}\right)$ represents an activity of about $1.3 \mathrm{MBq}$ (less than $0.5 \%$ of the injected ${ }^{18}$ F-FDG activity). Due to anatomical constraints not all activity is inside FOV of the detector. The random rate computed for patient $\mathrm{A}(f=0.47)$, and for a broad timing window (10 ns), was found to be $1.8 \mathrm{kHz}$ when no shielding is present in the setup. A reduction to $1.2 \mathrm{kHz}$ is achieved when the lead layer is protecting the detector heads from the patient torso single photon background. The NEC peak was found to be $0.38 \mathrm{kHz}$ and $0.42 \mathrm{kHz}$ without and with shielding respectively, both for a timing window of $4 \mathrm{~ns}$.

The maximum true rate for patient $\mathrm{B}$ increases comparatively to patient $\mathrm{A}(5.7 \mathrm{kHz})$. This increase is due to the smaller distance between the detector heads $(13 \mathrm{~cm})$ and to the volume of the breast being a factor of two larger $\left(1200 \mathrm{~cm}^{3}\right)$. The random rate $(10 \mathrm{~ns})$ is $5.0 \mathrm{kHz}$, reducing to 3.8 $\mathrm{kHz}$ when the shielding layer is present in the setup. In comparison with the previous case-study, the randoms rate has increased, even when the acceptance angle to events coming from outside the FOV is reduced by the smaller distance between the detector heads. Since the simulated activity in the patient torso is always the same, this means an increase of random coincidences originated in the breast. This is explained by a higher activity of about $2.5 \mathrm{MBq}$ (about $0.8 \%$ of the total injected activity into the patient) due to the enlarged breast volume. A NEC peak rate of $2.4 \mathrm{kHz}$ was achieved for a timing window of $4 \mathrm{~ns}$. For patient B exam, the presence of shielding gives only a marginally improvement of the NEC to $2.5 \mathrm{kHz}$. Although the true coincidence rate has increased, a larger fraction of randoms $(f=0.93$ computed for patient $\mathrm{B})$ contributes to the quality index of the data.

A $8.3 \mathrm{kHz}$ true rate was found for patient $\mathrm{C}$ breast exam scenario. The higher rate is a consequence of reducing the distance between the detector heads to $10 \mathrm{~cm}$ after breast compression has been applied to the patient. The breast compression will cause a more favorable distribution of the ${ }^{18}$ F-FDG activity throughout the FOV, bringing the breast volume into regions with higher sensitivities. For the same reason, the rate of random coincidences has slightly increased in comparison to patient $\mathrm{B}$, although the breast volume and simulated tissue activity are the same. The random rate $(10 \mathrm{~ns})$ was found to be $6.0 \mathrm{kHz}$ without shielding reducing to $5.0 \mathrm{kHz}$ in the presence of shielding. Regarding the NEC curve, the best performance is achieved for a timing window of 4-5 ns, with an absolute NEC value of $3.7 \mathrm{kHz}$. The presence of the lead layer, improves the NEC value to 4.2 $\mathrm{kHz}$.

For an energy window of 300-700 keV, scatter event rate do not have a significant effect on NEC, in spite of the fact that scatter rate increases for the largest breast volumes. The scatter fraction, calculated as $S F=S /(T+S)$, where $S$ represents the scattered event counts over the 


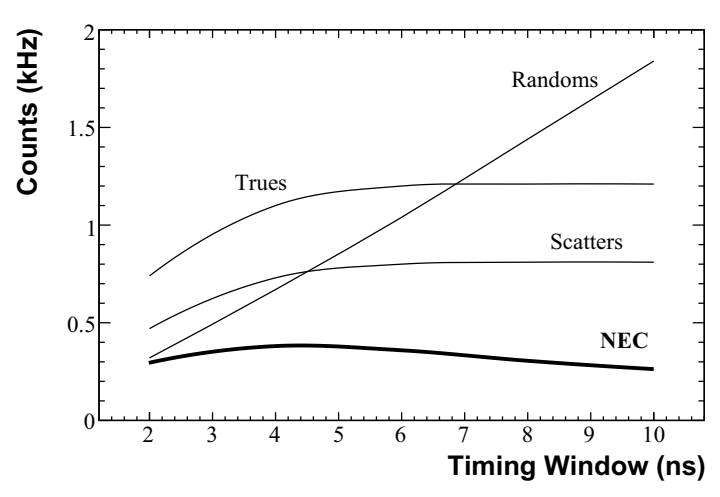

(a)

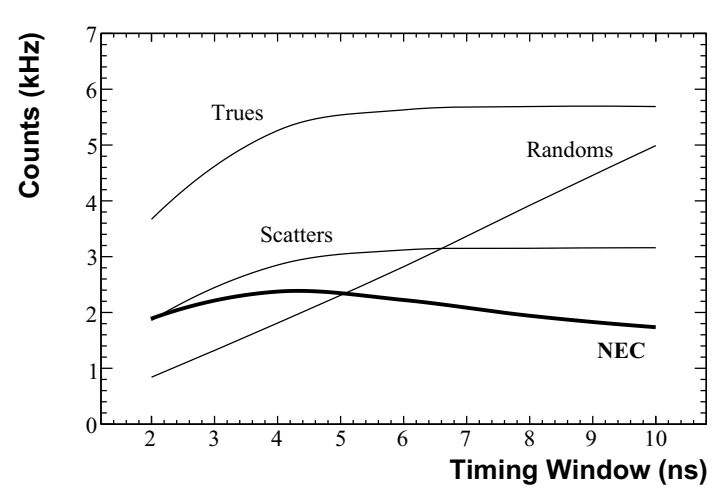

(c)

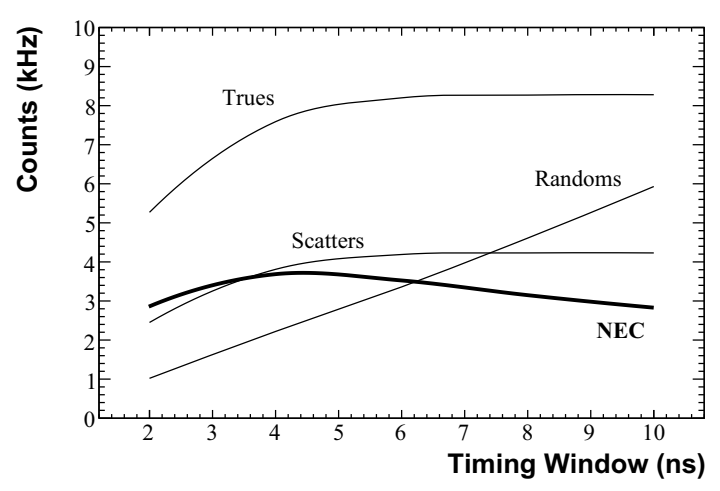

(e)

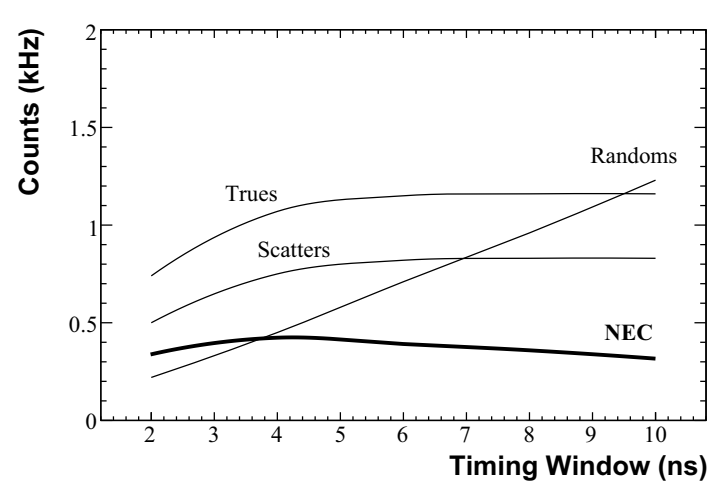

(b)

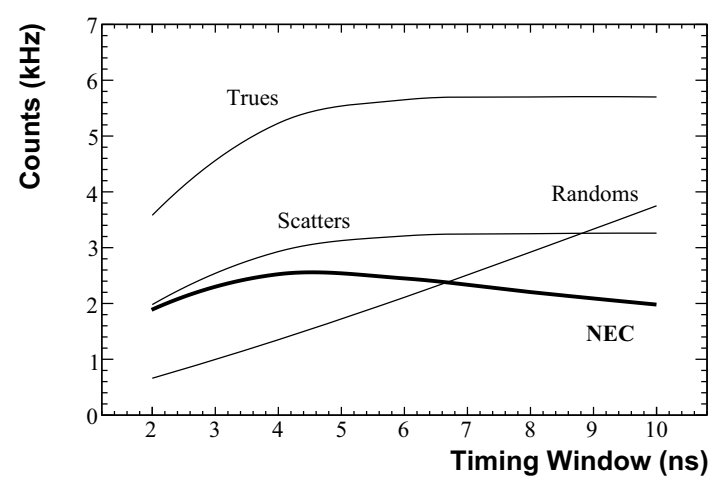

(d)

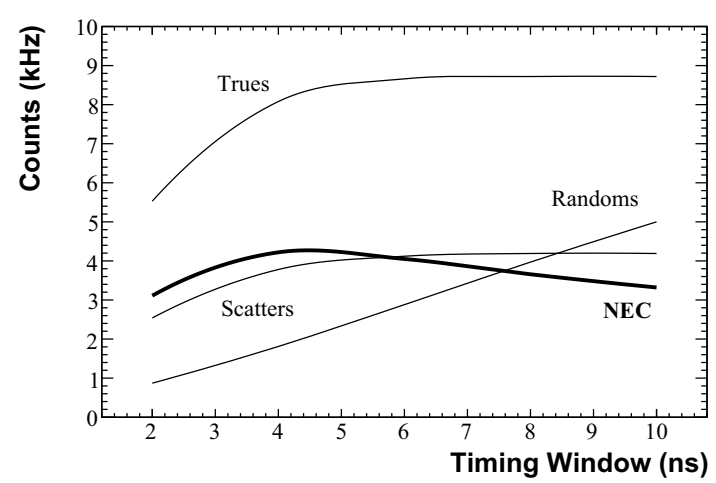

(f)

Figure 6. Counting rates for: patient $\mathrm{A}(\mathrm{a})+(\mathrm{b}), \mathrm{B}(\mathrm{c})+(\mathrm{d})$ and $\mathrm{C}(\mathrm{e})+(\mathrm{f})$ exam scenario with and without the presence of shielding in the simulated setup. 


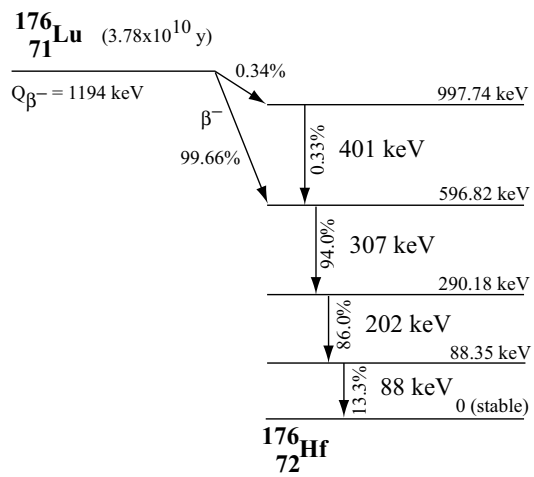

(a)

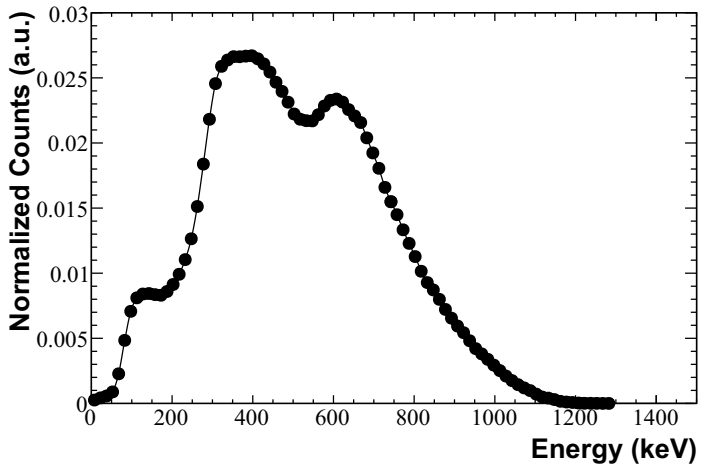

(b)

Figure 7. (a) Decay scheme of ${ }^{176} \mathrm{Lu}$ (Lutetium) into ${ }^{176} \mathrm{Hf}$ (Hafnium) by $\beta^{-}$emission. (b) ${ }^{176} \mathrm{Lu}$ background spectrum in one LYSO:Ce isolated crystal with dimensions of $2 \times 2 \times 20 \mathrm{~mm}^{3}$. The three broad peaks correspond mainly to combinations between the $307 \mathrm{keV}, 202 \mathrm{keV}$ and $88 \mathrm{keV}$ photons with the emitted electron.

entire FOV and $T$ the unscattered true coincidences, was also evaluated for each exam scenario. A $30 \%$ scatter fraction was found for patient $\mathrm{A}$ and $\mathrm{B}$ and $28 \%$ for patient $\mathrm{C}$ (breast compression). For the evaluated case-studies, the dominant limiting factor affecting the NEC curves seems to be the random coincidence rate. Random rate is not only proportional to the timing window but it also increases with the square of the activity. Even in the presence of the shielding layer, the contribution of randoms is not reduced enough (mean reduction of 20\%) to cause a significant improvement of the NEC. These results suggest that, in order to improve NEC, random correction techniques which do not increase the noise contribution should be used. As an example, re-calculating the NEC rate for case-study related with patient $\mathrm{A}$, assuming a noise-free random correction technique $(k=1$ in equation 3.1), and increase of $22 \%$ of the NEC peak could be achieved. This is specially critical, when small breast volumes are being imaged as represented by patient A scenario.

\subsection{Effect of ${ }^{176}$ Lu natural background radiation}

The influence of LYSO:Ce crystals intrinsic natural activity on the system count rates and dead time estimates was also investigated. LYSO:Ce scintillator material is characterized by the presence of ${ }^{176} \mathrm{Lu}$ isotope with an abundance of $2.6 \%$ in the natural lutetium used in the crystals production. ${ }^{176} \mathrm{Lu}$ has a half-life of approximately $3.8 \times 10^{10}$ years and decays into ${ }^{176} \mathrm{Hf}$ by $\beta^{-}$emission, followed by nuclear de-excitation. Four photons with energies of $404 \mathrm{keV}, 307 \mathrm{keV}, 202 \mathrm{keV}$ and 88 $\mathrm{keV}$ are emitted - figure 7(a). De-excitation through the emission of atomic electrons by internal conversion also compete with the prompt photons. This decay is responsible for the presence of an additional background of singles events and as a consequence of random coincidences (such as $\beta \beta$ and $\beta \gamma \beta$ event topologies), within the FOV of the scanner. In addition, the simultaneous emission of a $\beta^{-}$within one crystal and the emission of a photon that can be detected by the opposite detector head after crossing the FOV, can mimic an ordinary true coincidence. From the image reconstruction point-of-view, these $\beta \gamma$ coincidences will provide inaccurate LORs with no relation 


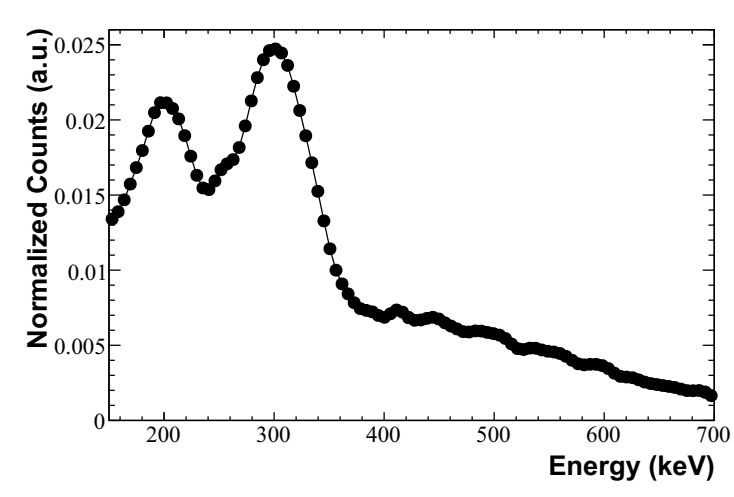

(a)

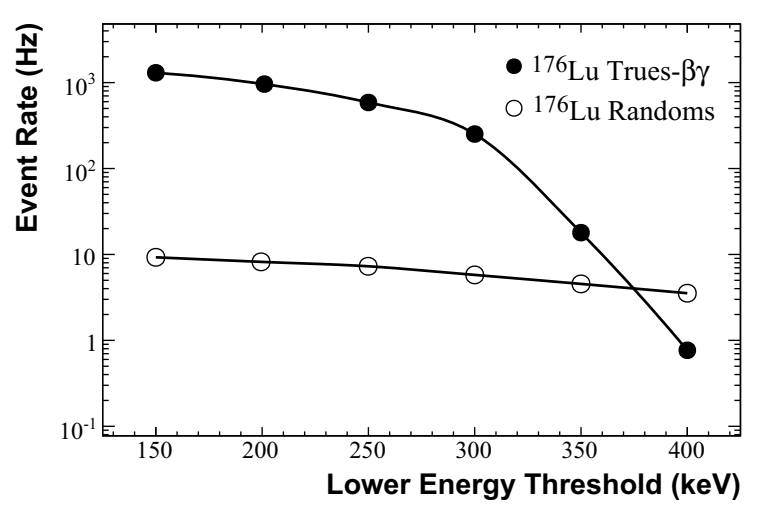

(b)

Figure 8. True $-\beta \gamma$ coincidences spectrum produced by the ${ }^{176} \mathrm{Lu}$ background. The two photoelectric peaks corresponds to coincidence events between $202 \mathrm{keV}$ and $303 \mathrm{keV}$ photons and a detected $\beta^{-}$. True $-\beta \gamma$ and random event rates as a function of the selected lower energy threshold recorded without activity within the FOV. The blank scan was simulated for a $10 \mathrm{~cm}$ separation distance between the detection heads.

with the activity in the FOV. The natural background activity is estimated at 240 Bq.cm ${ }^{-3}$ [20, 21]. The total volume of LYSO:Ce in the Clear-PEM scanner was calculated to be approximately 492 $\mathrm{cm}^{3}$, which results in an activity of $118 \mathrm{kBq}$ over the whole spectrum. The energy spectrum in one isolated LYSO:Ce crystal due to natural radiation obtained by simulation is shown in figure $7(\mathrm{~b})$,

In order to investigate the effect of the ${ }^{176} \mathrm{Lu}$ background, single, random and true- $\beta \gamma$ count rates after trigger simulation were assessed with the detector heads separated by $10 \mathrm{~cm}$. The obtained $\beta \gamma$ spectrum of coincidence events between emitted photons and a $\beta^{-}$detected in an opposing crystal within a timing window of $4 \mathrm{~ns}$ is shown in figure 8(a). Two photopeaks with energies around $200 \mathrm{keV}$ and $300 \mathrm{keV}$ can be observed in addition to a high energy tail extended over the region of the $511 \mathrm{keV}{ }^{18} \mathrm{~F}$ photopeak. This represents a background contamination for the full ${ }^{18} \mathrm{~F}$-FDG coincidence spectrum, which impact can be minimized by selecting an adequate lower energy threshold. True counts rates due to $\beta \gamma$ coincidences as well as the random coincidence rate due to LYSO:Ce intrinsic activity for a fixed timing window of $6 \mathrm{~ns}$ as a function of the energy threshold, are shown in figures $8(\mathrm{~b})$. The $\beta \gamma$ rate decreases as the lower energy threshold increases, since more true coincidences are rejected. For the wide-open energy window the true event rate was found to be $1.3 \mathrm{kHz}$, that represents about $8 \%$ of the ${ }^{18} \mathrm{~F}-\mathrm{FDG}$ true rate previously estimated for patient $\mathrm{C}$ exam. Above $300 \mathrm{keV}$ the count rates fall-off rapidly and for a lower energy threshold of $350 \mathrm{keV}$, the true event rate is already below $20 \mathrm{~Hz}$. The random count rate gradually decreases with the lower energy threshold and for a 150-700 keV energy window it was found to be $9.3 \mathrm{~Hz}$.

Simulation results suggest the use of a high energy threshold for the Clear-PEM scanner, in order to reject most part of the $\beta \gamma$ background. The impact of the background on the performance indicators previously obtained for the breast exam of patient $\mathrm{C}$ has been also evaluated. The NEC rate was re-calculated taking into account the true $-\beta \gamma$ and random coincidences from LYSO:Ce as [22]:

$$
N E C=\frac{T^{2}}{(T+S+L Y S O)+k f R}
$$


where, $T$ and $S$ are the total number of true and scattered events detected within the FOV. LYSO is the true $-\beta \gamma$ count rate produced by the ${ }^{176} \mathrm{Lu}$ background and $R$ is the random coincidence rate. The random rate includes the additional contribution of single events from the LYSO:Ce natural radioactivity to the total rate. Factors $f$ and $k$ were assumed to be unchanged and were set to 0.93 and 2 respectively. The peak NEC decreases only $4.5 \%$ for a lower energy threshold of both $150 \mathrm{keV}$ and $300 \mathrm{keV}$. These results do not represent a dramatically degradation of the data quality index and thus the ${ }^{176} \mathrm{Lu}$ background is not considered a constraining factor for Clear-PEM. Nevertheless, for smaller FOVs the detection sensitivity to $\beta \gamma$ and random coincidences is expected to be higher. For these reasons, the proposed settings of 300-700 keV energy window and 4-6 ns timing window, still seem to be the best compromise for the Clear-PEM scanner operation, between data quality for image reconstruction and detection efficiency.

\section{Discussion and conclusions}

Throughout this paper, Monte Carlo simulation was used to predict the counting rate performance of the Clear-PEM system. Realistic imaging conditions were simulated taking into account the influence of out-of-field activity due to ${ }^{18} \mathrm{~F}-\mathrm{FDG}$ uptake in the organs of an anthropomorphic patient model, a detailed description of the detector geometry and the modeling of data acquisition and trigger system response.

The proximity of the heart to the breast and the large volume of the liver were found to be two key factors for the high non-breast single photon background. The proposed lead shielding placed under the patient torso provides a moderate reduction of the background photons reaching the detector. The aperture for the breast was found to be the main cause of shielding inefficiency. The existence of higher single rates within trigger cells close to the patient torso do not represent a significant increase in the overall Clear-PEM system dead time. Under the simulated imaging conditions, the dead time estimates were found to be always below $5 \%$. For the $50 \mathrm{MHz}$ DAQ/Trigger baseline configuration, a maximum coincidence trigger rate of $670 \mathrm{kHz}$ is supported. For the investigated case-studies, a highest rate of $57 \mathrm{kHz}$ was found for the wide-open timing window of $10 \mathrm{~ns}$ and a lower energy threshold of $150 \mathrm{keV}$, ten-fold lower than the system limit. Radiotracer concentrations used in this studies were extracted from published data reported with large systematic uncertainties. Nevertheless, if one assumes an exam scenario where the activity concentrations within the different patient organs are a factor of two higher than the simulated ones, the total trigger rate will increase to $145 \mathrm{kHz}$ (the non-linear increase is due to the contribution from randoms), already corrected by a $9 \%$ system dead time due to the larger single flux reaching the detector front-end. In this case, the total trigger rate is still well within the bandwidth of the Clear-PEM DAQ/Trigger system. Regarding the data quality index, one can find that, in spite of the fact that random events increase with the square of the activity, the absolute NEC peak will still increase, from $3.7 \mathrm{kHz}$ to $5.2 \mathrm{kHz}$. This is indicative that NEC saturation point will only be reached for higher injected doses and uptake values expected in a typical breast exam. The present study also shows that ${ }^{176} \mathrm{Lu}$ background is not a constraining factor for the Clear-PEM scanner, leading to a negligible degradation of the system dead time and NEC rates. 


\section{Acknowledgments}

The authors would like to thank all members of the Crystal Clear Collaboration and also to acknowledge Fernando G. Almeida, João D. Aguiar and Filipe Lopes from Instituto de Engenharia Mecânica e Gestão Industrial (INEGI) and Faculdade de Engenharia da Universidade do Porto (FEUP) for providing us the Clear-PEM technical drawing. The Portuguese consortium PETMammography is financed by the Innovation Agency (AdI) and the Operational Program for Information Society (POSI), Portugal. The work of P. Rodrigues and A. Trindade is supported by Fundação para a Ciência e a Tecnologia (FCT) under grants 10187/2002 and 10198/2002.

\section{References}

[1] C.J. Thompsonet al., Positron Emission Mammography (PEM): a promising technique to detect breast cancer, IEEE Trans. Nucl. Sci. 42 (1995) 1012.

[2] W.W. Moses et al., PET camera designs for imaging breast cancer and axillary node involvement, J. Nucl. Med. 36 (1995) 69.

[3] E.L. Rosen et al., Detection of primary breast carcinoma with dedicated, large-field-of-view FDG PET Mammography device: initial experience, Radiology 234 (2005) 527.

[4] P. Lecoq et al., Clear PEM, A dedicated PET camera for mammography, Nucl. Instrum. Meth. A 486 (2002)1.

[5] M.C. Abreu et al., Design and evaluation of the Clear-PEM scanner for positron emission mammography, IEEE Trans. Nucl. Sci. 53 (2006) 71.

[6] R.R. Raylman et al., The potential role of positron emission mammography for detection of breast cancer. A phantom study, Med. Phys. 27 (2000) 1943.

[7] W.W Moses et al., Positron emission mammography imaging, Nucl. Instrum. Meth. A 525 (2004) 249.

[8] E. Albuquerque et al., The Clear-PEM electronics system, IEEE Trans. Nuc. Sci. 53 (2006) 2704.

[9] P. Bento et al, Performance simulation studies of the Clear-PEM DAQ/Trigger system, IEEE Trans. Nucl. Sci. 53 (2006) 2102.

[10] M. Rafecas et al., Inter-crystal scatter in a dual layer, high resolution LSO-APD positron emission tomography, Phys. Med. Biol. 48 (2003) 821.

[11] S. Agostinelli et al., Geant4-a simulation toolkit, Nucl. Instrum. Meth. A 506 (2003) 250.

[12] J. Allison et al., Geant4 developments and applications, IEEE Trans. Nucl. Sci. 53 (2006) 270.

[13] W.P. Segars, Development of a new dynamic NURBS-based cardiac torso (NCAT) phantom, PhD dissertation, The University of North Carolina (2001).

[14] The national laboratory of medicine's visible human project - segmented visible human data, http://www.brooks.af.mil/AFRL/HED/hedr/dosimetry.html/.

[15] R.R. Raylman et al., Corrections for the effects of accidental coincidences, Compton scatter, and object size in Positron Emission Mammography (PEM) imaging, IEEE Trans. Nucl. Sci. 48 (2001) 913.

[16] L. Zhang et al., Potential of a Compton camera for high performance scintimammography, Phys. Med. Biol. 49 (2004) 617. 
[17] Photon, electron, proton and neutron interaction data for body tissues, International Commission on Radiation Units and Measurements (ICRU) - Report 46 (1992).

[18] C. Ramos et al., FDG-PET standardized uptake values in normal anatomical structures using iterative reconstruction segmented attenuation correction and filtered back-projection, Eur. J. Nucl. Med. 28 (2001) 155.

[19] S.C. Strother et al., Measuring PET scanner sensitivity relating count rates to image signal-to-noise ratios using noise equivalent counts, IEEE Trans. Nucl. Sci. 37 (1990) 783.

[20] C.L. Melcher et al., Cerium-doped Lutetium Oxyorthosilicate: a fast, efficient new scintillator, IEEE Trans. Nucl. Sci. 39 (1992) 502.

[21] J.S. Huber et al., Effect of ${ }^{176}$ Lu background on singles transmission for LSO-based PET cameras, Phys. Med. Biol. 47 (2002) 3535.

[22] L. Eriksson et al., The ECAT HRRT: an example of NEMA scatter estimation issues for LSO-based PET systems, IEEE Trans. Nucl. Sci. 52 (2005) 90. 